\title{
The Adaptive Damping Technique: Improving the Simulation Accuracy of Hydraulic Transients
}

\author{
Jishnu Chandran R. \\ Department of Aerospace Engineering, \\ Indian Institute of Space Science and Technology, Thiruvananthapuram, Kerala, India. \\ Corresponding author: jishnuchandranr@gmail.com \\ A. Salih \\ Department of Aerospace Engineering, \\ Indian Institute of Space Science and Technology, Thiruvananthapuram, Kerala, India. \\ E-mail: salih@iist.ac.in
}

(Received on May 5, 2021; Accepted on September 30, 2021)

\begin{abstract}
Hydraulic surges are transient events frequently observed in various industrial and laboratory flow situations. Understanding surge physics and its accurate numerical prediction is crucial to the safety of flow systems. The maximum accuracy achievable for transient surge simulations is limited by the inefficiencies in the mathematical models used. In this work, we propose a mathematical model that incorporates an adaptive damping technique for the accurate prediction of hydraulic surges. This model also takes the compressibility effects in the liquid during the surge process into account. The novel approach of using the local pressure fluctuation data from the flow to adjust the unsteady friction for controlling the dissipation is introduced in this paper. The adaptive-dissipation is actualized through a unique 'variable pressure wave damping coefficient' function definition. Numerical simulation of three different valve-induced surge experiments demonstrates the reliability and robustness of the mathematical model. Numerical results from the proposed model show an excellent match with the experimental data by closely reproducing both the frequency and the amplitude of transient pressure oscillations. A comparative study explains the improvement in the simulation accuracy achieved by replacing the constant damping coefficient with the proposed variable coefficient. The superiority of the new model with the adaptive damping capability over the similar models in literature and those used in commercial software packages is also well established through this study.
\end{abstract}

Keywords- Adaptive damping, Hydraulic transient, Surge modelling, Unsteady friction, Variable damping coefficient.

\section{Introduction}

Transient variations in the fluid pressure at critical locations during valve closure operations in different flow systems are crucial to the safety of the respective systems and for selecting the optimal valve operating conditions. The complete understanding of a surge phenomenon requires the accurate estimation of frequency and amplitudes of pressure oscillations in the system, the time instants and locations at which they occur, and the time taken for these oscillations to dissipate. Most simulation techniques show improper modelling of pressure dissipation during hydraulic transients due to the limitations in the mathematical models used. Hence the mathematical model needs to be made more robust without any substantial rise in the computational cost.

The use of unsteady friction models that can adjust the amount of dissipation based on the local gradients in pressure is an effective way of improving the accuracy of simulations. Using the local pressure fluctuations for selective adjustment of the dissipation is a novel approach in surge modelling systems. A variable pressure wave damping coefficient (VPDC) is presented in the present work to achieve this adaptive damping in pressure surge simulation for valve-induced 
transients. Improvement in the simulation accuracy using this adaptive damping technique is investigated by modelling three different valve-closure experiments selected from the literature. Comparative performance analysis of this model is also presented using selected numerical data sets from the literature and using the simulation results from a commercial hydraulic surge analysis package called the 'AFT Impulse'.

The paper is organized as follows. In Section 2, important research contributions related to the present work are briefly reviewed, and the relevance of current research is corroborated. Section 3 provides details on the valve-closure experiments computationally solved in the present study. The mathematical model used for the simulation of hydraulic surges and the VPDC definition for the adaptive friction model is explained in Section 4. The computational strategy applied for the numerical simulation of surge problems is outlined in Section 5, along with a brief description of the AFT Impulse package. Section 6 of this paper present the main results and discusses the major observations and findings of the research. Section 7 briefly concludes the research work.

\section{Literature Review and Relevance of the Research}

Valve-induced hydraulic surges is an area of extensive research and have eventually led to numerous experimental and computational studies in this field. Some of the notable experimental studies on valve-induced surges were carried out by Covas et al. (2004), Balacco et al. (2015), and Kodura (2016). Computational studies by Ramos et al. (2004), Covas et al. (2005), and Nikpour et al. (2014) critically examined the impact of sudden valve closures on the generation of hydraulic transients. Tran (2017) and Lai et al. (2018) numerically explored the characteristics of pressure transients induced by fast valve closures in pipelines. Controlled fluid transients are also used as fault and leak detection techniques for pipelines, and a review of this technique is presented by Colombo et al. (2009) and recent works by Bohorquez et al. (2020) and Wang et al. (2020) reports the application of this technique. A three-dimensional simulation of valve-induced water hammer was presented by Yang et al. (2017). Wang et al. (2018) investigated on the role of friction models in the simulation of transients arising during pipeline filling. Firkowski et al. (2019) studied the influence of unsteady friction on the simulation results for pressure transients in viscoelastic pipes. This study also suggested the need for a dimensionless parameter that can help to decide on the suitable friction model for a given transient simulation. Different families of unsteady friction models were compared by Mandair et al. (2020) for the simulation of water hammer problem by using an integrated total energy approach. A recent study by Abdeldayem et al. (2021) investigated the performance of different unsteady friction models for their suitability to practical transient simulations and implemented some of these in the WANDA commercial software.

Use of adaptive friction models is an effective way of achieving computationally inexpensive and high accuracy simulations. Chandran et al. (2020) presented an unsteady friction model with a variable pressure wave damping coefficient (VPDC) for simulation of the surges downstream of a closing valve. In the present work, we intend to extend this model for the numerical simulation of valve-induced transients at the upstream location of closing valves. The VPDC needs to be defined in a simple form that could make it easy to incorporate it into different mathematical models and commercial solvers. A well-controlled dissipation of pressure is required from the adaptive friction model to improve the accuracy of simulations and the numerical stability of solvers. The suitability and consistency of the adaptive-friction model for surge modelling are to be verified by applying it to model varied flow conditions and operating ranges. 


\section{Details of Valve-Closure Experiments Selected for the Numerical Study}

Characteristics of a hydraulic surge depend on many geometrical, structural and flow parameters. For this work, we have selected three different valve induced hydraulic surge experiments from the literature that involve different pipe materials, wave speeds, geometrical sizes, and operating pressure ranges. The first two experiments are by Mitosek and Szymkiewicz (2012), where the first one (Mitosek Exp-1) was conducted with a steel pipeline and the second experiment (Mitosek Exp2) used a high-density polyethylene (HDPE) pipe. The third experiment by Soares et al. (2015) used a copper pipeline. The values of various experimental parameters are provided in Table 1.

Table 1. Details of experiments.

\begin{tabular}{|c|c|c|c|}
\hline \multirow{2}{*}{ Parameters } & \multicolumn{2}{|c|}{ Experiment } & Soares et al. (2015) \\
\cline { 2 - 4 } & $\begin{array}{c}\text { Mitosek and } \\
\text { Szymkiewicz (2012)-1 }\end{array}$ & $\begin{array}{c}\text { Mitosek and Szymkiewicz } \\
\mathbf{( 2 0 1 2 ) - 2}\end{array}$ & Copper \\
\hline Pipe Material & Steel & HDPE & 15.22 \\
\hline Pipe Length [m] & 72.0 & 240.0 & 0.020 \\
\hline Pipe diamter [m] & 0.042 & 0.0352 & 0.0010 \\
\hline Pipe thickness [m] & 0.0033 & 0.0024 & 300 \\
\hline System temperature [K] & 287.15 & 287.15 & 0.46 \\
\hline System head [m of H2O] & 51.0 & 23.1 & 1255 \\
\hline Flow velocity [m/s] & 0.41 & 0.86 & $16.5 \times 10^{-3}$ \\
\hline Wave speed [m/s] & 1245 & 335 & 0.5 \\
\hline Valve closure duration [s] & $25 \times 10^{-3}$ & $20 \times 10^{-3}$ & 32.0 \\
\hline Experiment duration [s] & 8.0 & & \\
\hline
\end{tabular}

All the experiments considered here are pure single-phase flow cases without cavitation effects. Experimentally measured transient pressure data are available for each of these experiments at the upstream location of the closing valve. We intend to numerically simulate these surge cases using the adaptive friction integrated mathematical model with the respective geometrical and flow conditions. The predicted numerical surge data will be analyzed and validated against the respective experimental data.

\section{The Mathematical Model}

A suitable mathematical model for the representation of physical laws that govern the flow transient is selected. Further, the definition of a VPDC and its incorporation into the unsteady friction formulation of the mathematical model is elaborated.

\subsection{Governing Relations - The Two-Equation Compressible-Liquid Model}

The two-equation compressible-liquid model proposed by Chandran et al. (2020) is adopted for simulating the selected transient flow cases. The important governing relations for the flow in this model, viz., the fluid mass balance and the momentum balance, are given as follows:

$$
\begin{aligned}
& \frac{1}{a^{2}} \frac{\partial p}{\partial t}+\frac{u}{a^{2}} \frac{\partial p}{\partial x}+\rho \frac{\partial u}{\partial x}=0 \\
& \frac{\partial u}{\partial t}+u \frac{\partial u}{\partial x}+\frac{1}{\rho} \frac{\partial p}{\partial x}=-\frac{4 \tau}{\rho d}
\end{aligned}
$$

The system is assumed to be at isothermal conditions and thus the energy equation need not be solved explicitly. Therefore, we have a system of PDEs with $u, p$, and $\rho$ as the dependent variables. Transient variations in both signal celerity and dissipation of pressure waves are common for valve- 
induced transients. These variations are generally not accurately reproduced by the standard unsteady pipe flow models. A strategy to account for the time-varying celerity of the pressure waves is to model the compressibility effects in the liquid. The modified NASG EOS proposed by Chandran and Salih (2019) is used to model the compressibility effects of water. Density of the liquid is calculated as the reciprocal of its specific volume, which is given by the following relation of the modified NASG EOS:

$$
v=\frac{(\gamma-1) C_{V} T}{\left(p+P_{\infty}\right)}+b
$$

The speed of sound in the unconfined liquid is given by the following relation:

$$
c=\sqrt{\frac{\gamma v^{2}\left(p+P_{\infty}\right)}{v-b}}
$$

The actual signal propagation speed in the system is estimated from the speed of sound in the liquid, and the geometric dimensions and elastic properties of the pipe, as follows:

$$
a=\frac{1}{\sqrt{\frac{1}{c^{2}}+\left(1-v^{2}\right) \frac{\rho d}{E s}}}
$$

Estimation of shear stress is based on the unsteady friction model presented in Section 4.2.

\subsection{Friction Model- The Variable Pressure Wave Damping Coefficient}

Friction models play a crucial role in the simulation of highly transient flow problems. Drastic variations in flow properties during the initial phase of a transient is quickly dissipated within the system to attain steady-state conditions. Robust friction models are required to accurately simulate these transient variations in flow variables. Simulation of highly transient cases with the commonly used steady or unsteady friction models can lead to non-physical results. This is because of the incapability of such models to adapt to the varying dissipation requirements in the system based on time and space. One could develop or modify an existing unsteady friction model for higher simulation accuracy by improving its capability to better adapt to the varying flow requirements.

Chandran et al. (2020) suggested replacing the constant damping coefficient $k$ in the unsteady friction model with a variable pressure wave damping coefficient $k_{v d}$. Parameter $k_{v d}$ is specifically defined to improve the accuracy of the surge prediction at locations downstream of the closing valve. However, the surge characteristics at the upstream location of a closing valve are different from those at the downstream side. Therefore, similar to $k_{v d}$, we need to define a new variable pressure wave damping coefficient, $k_{v u}$, for surge modelling at the upstream side of a closing valve. The variable parameter, $k_{v u}$ is defined as a function of the parameter 'relative local fluctuation of pressure', $\Delta P_{R L F}$. This non-dimensional parameter is the ratio of 'magnitude of the local pressure fluctuations' $\left(\left|p_{i}-p_{0}\right|\right)$ to the 'maximum possible pressure fluctuation' $\left(\Delta P_{\max }\right)$. The new variable pressure wave damping coefficient is defined as follows:

$$
k_{v u}=m_{1}\left[\Delta P_{R L F}\right]^{m_{2}}=m_{1}\left[\frac{\left|p_{i}-p_{0}\right|}{\Delta P_{\max }}\right]^{m_{2}}
$$

In equation (6), $m_{1}$ and $m_{2}$ are tunable parameters. The parameter $m_{1}$ controls the overall dissipation and $m_{2}$ provides fine-tuning for the dissipating trend of the transient. Higher dissipation 
is achieved for large values of $m_{1}$ and vice-versa. Generally, numerical models are observed to accurately predict the initial high peaks of transients. Excess dissipation observed in the numerical results at later stages of transients is avoided by the parameter $\Delta P_{R L F}$. This is because, it lowers the value of $k_{v u}$ for small scale fluctuations in pressure from operating conditions. With this definition of $k_{v u}$, the shear stress model used by Neuhaus and Dudlik (2006) is rewritten as follows:

$$
\tau=\frac{f}{4} \frac{\rho}{2} u|u|+\left(k_{v u} \frac{\rho d}{4} a\right) \operatorname{sign}(u)\left|\frac{\partial u}{\partial x}\right|
$$

Source term in equation (2) is computed using the shear stress estimated from equation (7).

\section{The Computational Strategy}

Numerical simulations of all the cases are carried out using a generalized one-dimensional experimental layout with a straight pipe assumption as shown in Figure 1.

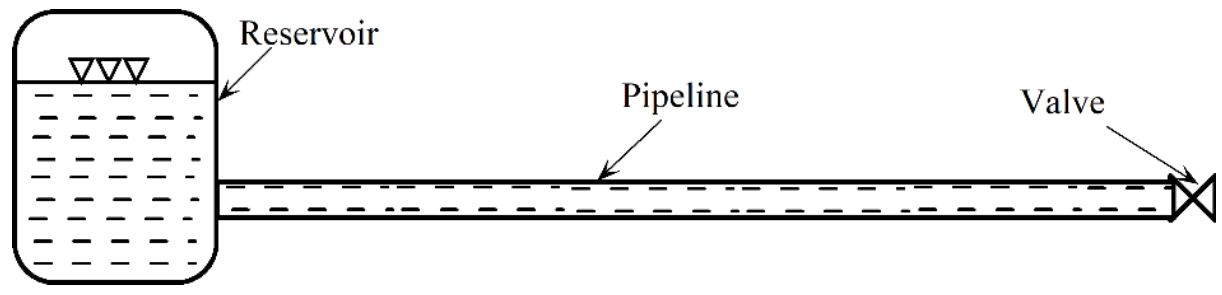

Figure 1. The generalized layout for experiments.

A one-dimensional approach is used here because the variations in the flow variables for valveinduced hydraulic transients are generally unidirectional. The setup consists of a straight pipeline carrying fluid (liquid water) from a pressurized reservoir and connected with a fast-closing valve. We intend to numerically compute the transient pressure variation at the location just upstream of the closing valve for all the experiments considered. On the left boundary, a fixed pressure inlet condition is assigned to represent the flow from the constant pressure reservoir. Valve closure at the right boundary is modelled by specifying a linearly decreasing velocity boundary condition that lasts over a few milliseconds. Beyond this duration, the velocity at the right boundary is maintained as zero to emulate the completely closed valve. Velocity at the left boundary and pressure at the right boundary are extrapolated from the property values within the domain.

The computational algorithm used is the one presented by Chandran et al. (2020) and validated using the experimental data. After the conversion of governing equations into their matrix form, a two-step solution algorithm was used. On defining the characteristic vector $W$ such that:

$$
W=\left[\begin{array}{c}
-\frac{p}{2 \rho a}+\frac{u}{2} \\
\frac{p}{2 \rho a}+\frac{u}{2}
\end{array}\right]
$$

The characteristic form of the equation excluding the source term becomes:

$$
\frac{\partial W}{\partial t}+\wedge \frac{\partial W}{\partial x}=0
$$


In the first step, using the Split coefficient matrix (SCM) method, the eigenvalue matrix is split into respective characteristic speed matrices and equation (9) is solved for an intermediate value denoted by ' $\star$ ' using data from the $n^{\text {th }}$ time step as follows:

$$
W_{i}^{\star}=W_{i}^{n}-\frac{\Delta t}{\Delta x}\left[\Lambda^{+}\left(\frac{\partial W}{\partial x}\right)^{+}+\Lambda^{-}\left(\frac{\partial W}{\partial x}\right)^{-}\right]_{i}^{n}
$$

This intermediate $\star^{\prime}$ 'values do not include the effects of source term. The matrix of eigenvalues, $\Lambda$ is split into the characteristic speed matrices with positive eigenvalues, $\Lambda^{+}$and negative eigenvalues, $\wedge^{-}$. Convective terms in equation (10) are discretized using an explicit third-order upwind method. The primitive $\star^{\prime}$ variables are computed from the characteristic ' $\star^{\prime}$ variables. In the second step, the effects of the source term are included in the solution by solving the transient part of the governing relations with the source term. Source terms are absent in the mass conservation relation, and therefore the source term integration step requires updating only the momentum relation. This step corresponds to updating the flow velocity as follows:

$$
u_{i}^{n+1}=u_{i}^{\star}-\Delta t\left(\frac{4 \tau}{\rho d}\right)
$$

\subsection{Hydraulic Surge Modelling using the AFT Impulse Software}

'Impulse' is a method of characteristics (MOC) based surge analysis software package developed by the Applied Flow Technologies (AFT) Pvt. Ltd. The selected valve-closure experiments were simulated using the 'AFT Impulse 8' package. This package offers steady and unsteady friction models, different valve types, and various transient valve-closure functions. The experimental setup for valve-induced surges in the Impulse platform is as shown in Figure 2.

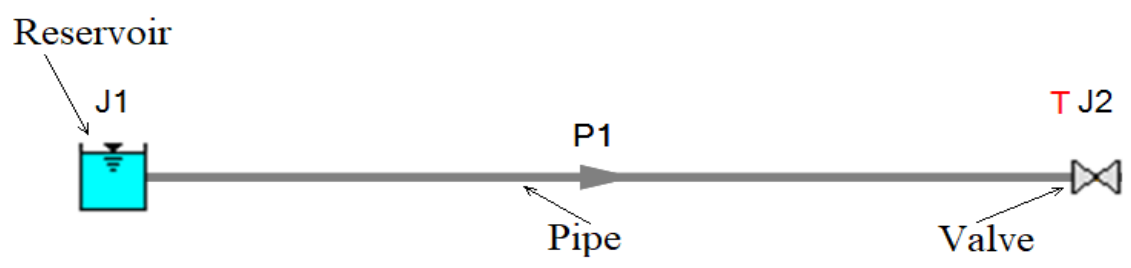

Figure 2. The generalized layout for experiments.

The pipe section is defined using details such as pipe's material, length, diameter, thickness etc. The system automatically calculates the wave speed in the domain. The initial volume flow rate is input for each case, and fluid properties such as density, dynamic viscosity, vapour pressure, bulk modulus, etc., are obtained from the NIST REFPROP database. A ball-type valve with a transient closure condition over a preset duration is assigned at the right boundary. The reservoir at the left boundary is specified by assigning values for the fixed liquid surface pressure, level of water above the pipe center, and height of the system from the reference.

The friction model is specified using the 'explicit friction factor' option under the 'user-specified' tab. The Darcy friction factor based on the respective flow Reynolds number was calculated and assigned to the solver. 'Variable pipe resistance' option was also enabled for all the simulations performed. Impulse offers steady, unsteady and advanced unsteady (Bruonne) friction models. The valve-transient was assigned as a linear decrease of the flow coefficient of the valve, $\mathrm{K}_{\mathrm{v}}$, from the 
full open value down to zero over the duration of valve closure. The sectioning of pipes was carried out based on the suggestion from the MOC-based solver. The Grid-independency of numerical results was also checked and confirmed. Results are obtained for pipes at their inlet, outlet, and at all intermediate stations.

\section{Results and Discussion}

The three valve-induced surge cases are numerically simulated using the proposed mathematical model that incorporates the adaptive damping technique. Numerical pressure profiles at the upstream location of the valve for Mitosek's experiment on steel pipe is displayed in Figure 3. Numerical solution data from the two-equation compressible model with the constant and the variable damping coefficients, solution profiles from the AFT Impulse software with different unsteady friction models are also shown in this figure. Figure 3 also displays the experimental data to compare the performance and accuracy of these computational techniques.

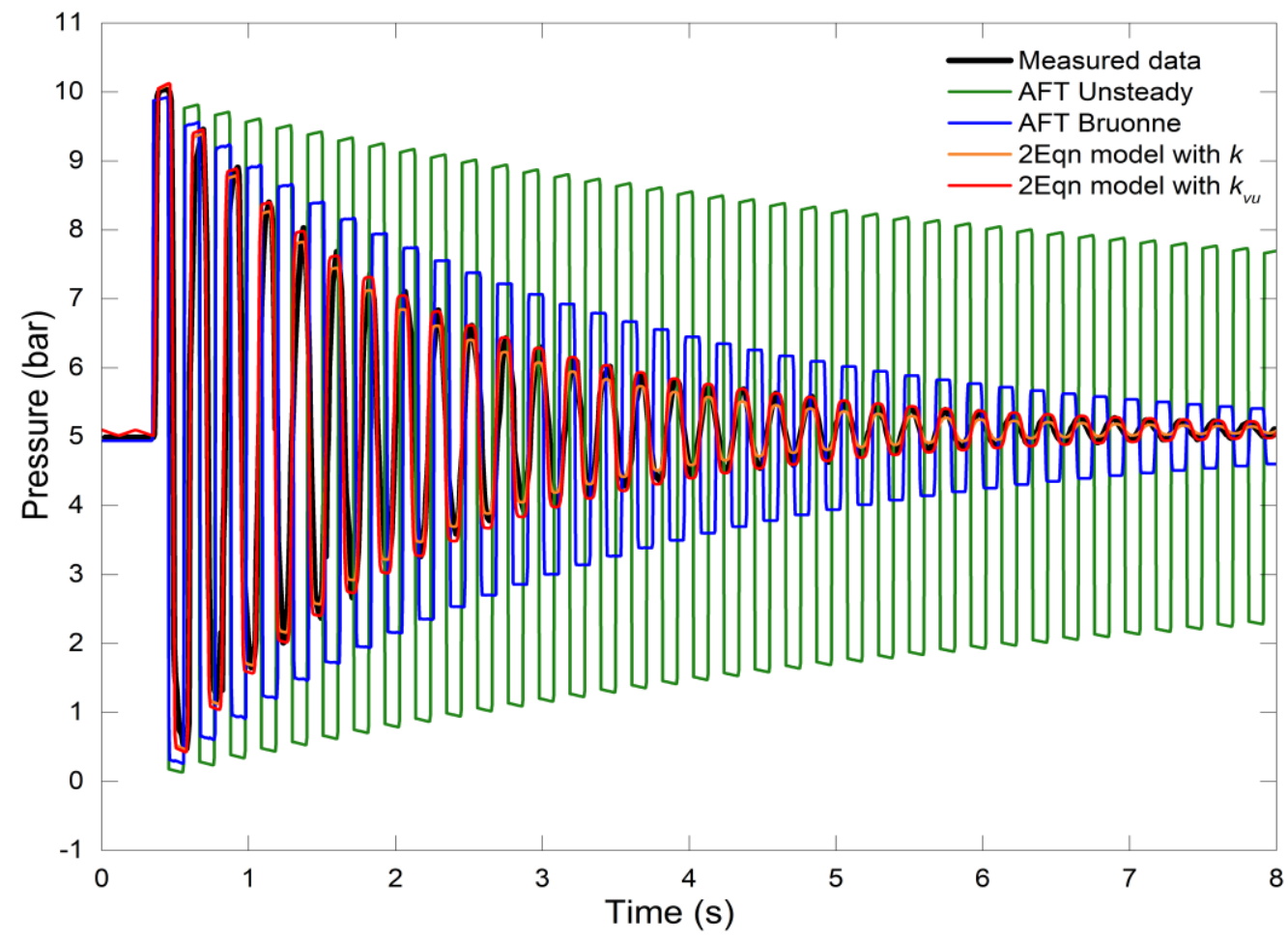

Figure 3. Transient pressure profiles for Mitosek's experiment on steel pipe predicted by different models.

The initial peaks of the transient surge are accurately captured in the numerical solutions. However, with the progress of the transient, dissipation in the numerical pressure data is found to be insufficient compared to the experimental data. Friction factors calculated using the standard relations are unable to produce expected damping in the pressure profile. For Mitosek et al.'s experiment on steel pipe, AFT unsteady and advanced unsteady (Bruonne) friction models show a difference in the numerical pressure profiles. In this case, the Bruonne model offers higher dissipation and better accuracy in the numerical results. Solution profiles from Impulse 8 also show a higher frequency for pressure oscillations. 
Numerical pressure profiles from the two-equation model are in close agreement with the measured values. They are distinctively superior to the results from AFT Impulse. The two-equation model with the VPDC, $k_{v u}$ produced results nearest to the field data, while the same model with a constant $k$ shows certain deviations. The numerical profile corresponding to a constant $k$ in Figure 3 clearly shows extra dissipation, which is persistent throughout the transient. The inclusion of VPDC into the model has removed this unwanted dissipation from the solution profiles. Amplitude and frequency of the pressure oscillations predicted with the mathematical model incorporating the adaptive friction formulation exhibit a close match with experimental data. This fine match between the predicted results and the field data is maintained at all stages of the transient.

Numerical solution profiles for Mitosek et al.'s experiment on HDPE pipe is displayed in Figure 4. Solution profiles from the two-equation model are compared against the experimental data, simulation results from AFT Impulse, and that obtained using the unsteady friction model of Ramos et al. (2004). No differences are observed in the results generated using the unsteady and the advanced unsteady friction models of AFT Impulse for the surge in HDPE pipe.

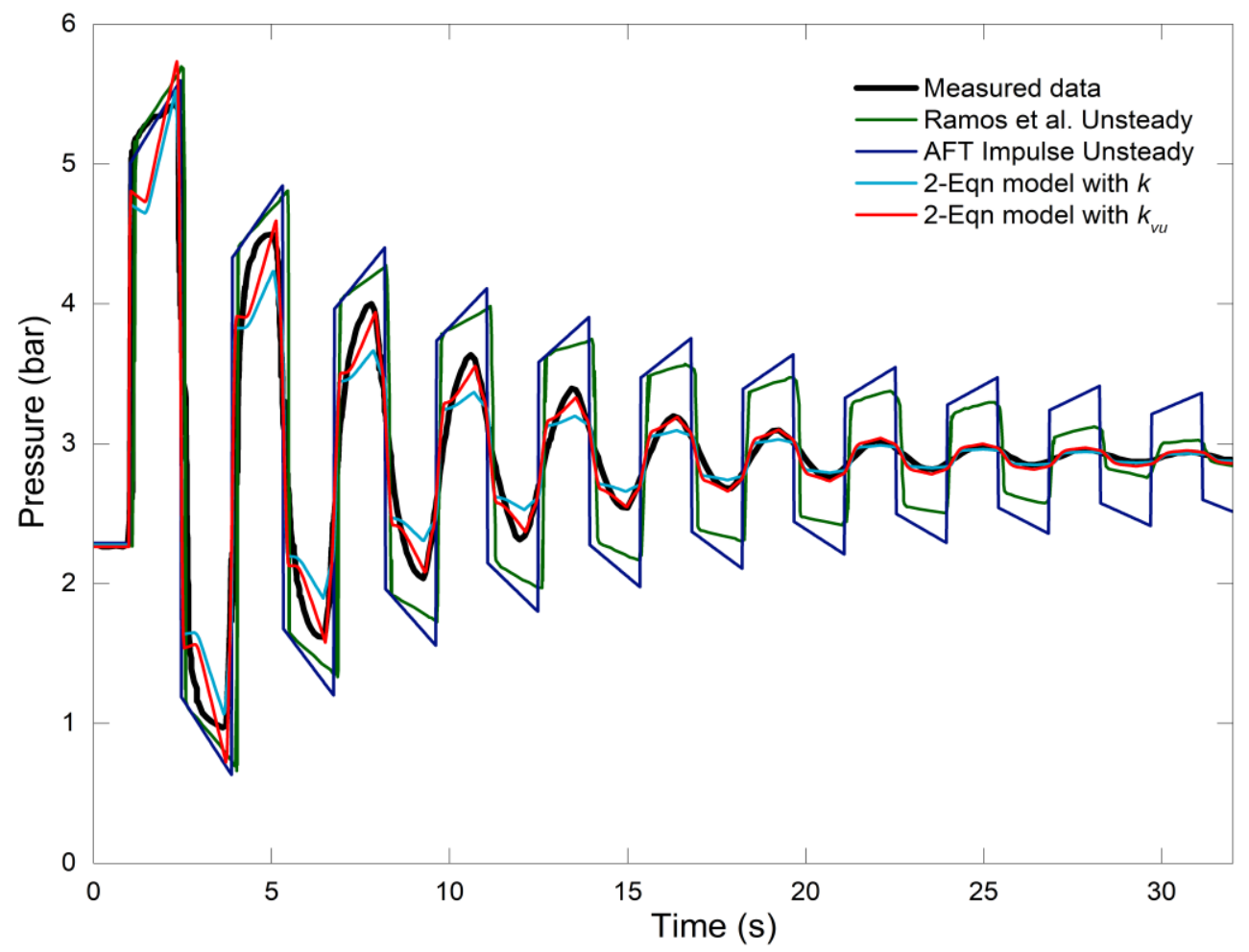

Figure 4. Transient pressure for Mitosek's experiment on HDPE pipe predicted by different models.

Results from Impulse 8 show a decaying wave pattern that is considerably over-predicted in comparison to experimental data. Unsteady model of Ramos et al. exhibits better dissipation in the solution profile and is superior to Impulse's unsteady friction model. However, this model also shows notable deviations from field data. The two-equation model is the most accurate method and the solution from this model are in good agreement with the field data. All models predict the pressure oscillation frequency with reasonable accuracy. The fixed amount of damping from the 
constant damping coefficient proves to be excessive for a particular phase of the transient while it appears insufficient for some other phase. The variable damping coefficient addresses these deficiencies to a convincing extent and provides accurate damping with respect to the measured data.

Figure 5 shows the transient pressure profiles for Soares' experiment on the copper pipe. The numerical solution from the two-equation model for this experiment is compared against the experimental data and the simulation results from AFT Impulse. Though the frequency of pressure oscillations is accurately predicted by the Impulse platform, the decay of these oscillations is not satisfactorily reproduced by the software due to insufficient damping. The two-equation model with adaptive damping again produced a superior solution that closely follows the experimental data. The adaptive dissipation achieved in the pressure profile through the incorporation of VPDC into the two-equation model is visible in the solution plot in Figure 5.

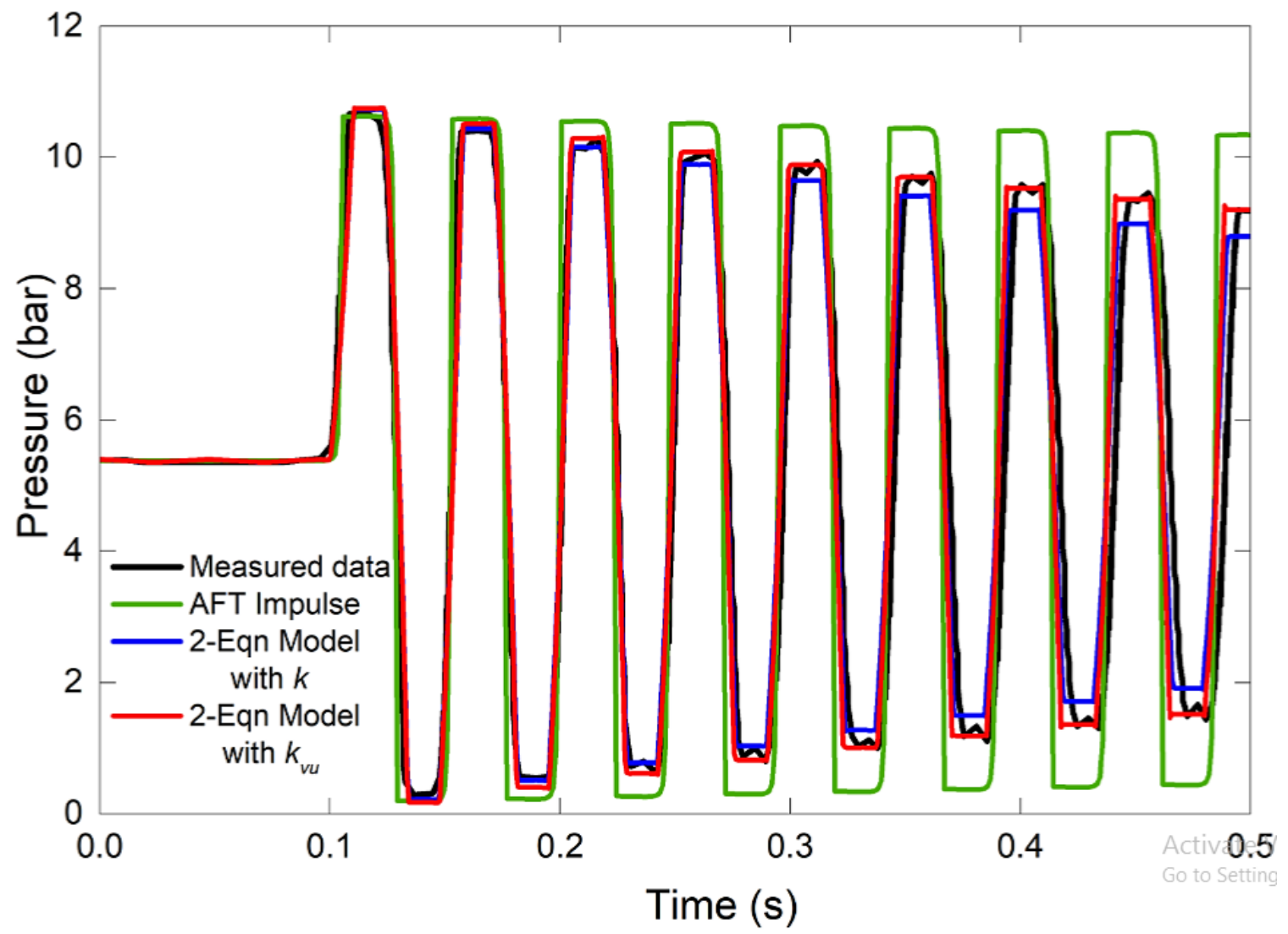

Figure 5. Transient pressure profiles for Soares' experiment on copper pipe predicted by different models.

The extra dissipation observed with a constant $k$ is rectified by using the variable $k_{v u}$ instead. Though the solution profiles obtained using $k_{v u}$ are in close agreement with experimental profiles, a small phase shift is observed to develop between the numerical and the experimental profiles as the transient progress. This observation can be attributed to the variations in the signal speed estimates. 
The fixed value of pressure damping coefficient, $k$, applied throughout the computation makes it inflexible to adjust based on varying flow conditions. Flexibility added to the mathematical model by using the VPDC, $k_{v u}$, to predict numerical results close to measured data is notable specifically towards the end phase of the transients. The optimal values for constant damping coefficient, $k$, for Mitosek's experiments respectively on Steel and HDPE pipes are 0.07 and 0.17, and for the Soares' case this value is observed to be 0.025 . The most accurate numerical solution is obtained when the value of parameter $m_{1}$ in the VPDC function definition is maintained at the respective value of $k$ for that particular experiment. This is because for all the cases considered we could observe a general trend that the two-equation model with constant $k$ closely predicts the initial peak of pressure surge and only at the later phase of transient, the dissipation becomes excessive. Thus the parameter $m_{1}$ responsible for the overall damping could be assigned the same value as $k$. However, to avoid the extra dissipation occurring later on in the transient, the parameter $m_{2}$ needs optimization. Optimal values of $m_{2}$ for the experiments are found to be in the range of 0.1-0.3.

The simplified assumption of elliptically looped pipe in Soares' experiment as a straight pipe for one-dimensional modelling could be a possible reason for any mismatches between the numerical and experimental data. The proposed mathematical model equipped with the adaptive damping capability from the unique VPDC function definition accurately predicted the transient pressure profiles for all three cases studied and demonstrated its reliability.

\begin{tabular}{|c|c|c|c|}
\hline Alphabets & & Greek Symbols & \\
\hline$a$ & Signal propagation speed & $\rho$ & Density \\
\hline$b$ & Covolume of liquid & $\gamma$ & Ratio of specific heats \\
\hline$c$ & Sound speed in medium & $\tau$ & Shear stress \\
\hline$C_{V}$ & Isochoric specific heat & $\vartheta$ & Poisson's ratio \\
\hline$d$ & Diameter of pipe & $\Lambda$ & Matrix of Eigen values \\
\hline$E$ & Young's Modulus & & \\
\hline$f$ & Darcy friction factor & Abbreviations & \\
\hline$k$ & Constant pressure wave damping coefficient & AFT & Applied Flow Technology \\
\hline$k_{v d}$ & $\begin{array}{l}\text { Variable pressure wave damping coefficient for } \\
\text { downstream surges }\end{array}$ & HDPE & High density poly ethylene \\
\hline$k_{v u}$ & $\begin{array}{l}\text { Variable pressure wave damping coefficient for } \\
\text { upstream surges }\end{array}$ & MOC & Method of Characteristics \\
\hline$m_{1}$ & Parameter responsible for overall damping & NIST & $\begin{array}{l}\text { National Institute of Standards and } \\
\text { technology }\end{array}$ \\
\hline$m_{2}$ & Parameter responsible for overall damping trend & VPDC & $\begin{array}{l}\text { Variable pressure wave damping } \\
\text { coefficient }\end{array}$ \\
\hline$p$ & Pressure & & \\
\hline$P_{\infty}$ & Stiffening parameter & Superscripts & \\
\hline$\Delta P_{\max }$ & Maximum fluctuation in pressure & + & Positive Eigen values \\
\hline$\Delta P_{R L F}$ & Relative local fluctuation in pressure & - & Negative Eigen values \\
\hline$t$ & Temporal co-ordinate & $*$ & Intermediate state \\
\hline$T$ & Temperature & $n$ & Time level index \\
\hline$u$ & Horizontal component of velocity & & \\
\hline$v$ & Specific volume & Subscripts & \\
\hline$W$ & Characteristic variable vector & 0 & Reference value \\
\hline$x$ & Spatial co-ordinate & $i$ & Spatial cell index \\
\hline
\end{tabular}




\section{Conclusions}

The difference in the transient trends in surge data on either side of a closing valve necessitated a new definition for VPDC for accurate surge prediction at upstream locations of a closing valve. Selected data sets from literature and those from AFT Impulse software exhibit insufficient damping of pressure oscillations. The two-equation model was used to numerically simulate three different valve-induced hydraulic transients. Though the two-equation model offers comparatively better solutions, the dissipation becomes excessive as the transient progresses. Replacement of the constant damping coefficient with the VPDC brings considerable improvement in simulation accuracy. The unique function definition of VPDC provides adaptive damping capability to the numerical solver, and the results obtained using it shows a close match with the experimental data. The proposed model used the novel approach of calculating the local fluctuations in pressure and using this data to suitably adjust the dissipation to achieve accurate estimation of the pressure oscillations. The VPDC definition having a lucid form can be easily incorporated into more mathematical models and commercial solvers. Also, by performing more experiments, the tunable parameters in the VPDC definition need to be developed into a function of flow variables. The controlled dissipation provided by this adaptive damping technique can improve the simulation accuracy and numerical stability of solvers to which it is incorporated.

\section{Conflict of Interest}

The authors declare that they have no conflict of interest.

\section{Acknowledgments}

This research did not receive any specific grant from funding agencies in the public, commercial, or not-for-profit sectors. The authors would like to thank the editor and anonymous reviewers for their comments that help improve the quality of this work.

\section{References}

Abdeldayem, O.M., Ferràs, D., van der Zwan, S., \& Kennedy, M. (2021). Analysis of unsteady friction models used in engineering software for water hammer analysis: implementation case in WANDA. Water, 13(4), 495.

Balacco, G., Apollonio, C., \& Piccinni, A.F. (2015). Experimental analysis of air valve behaviour during hydraulic transients. Journal of Applied Water Engineering and Research, 3(1), 3-11.

Bohorquez, J., Alexander, B., Simpson, A.R., \& Lambert, M.F. (2020). Leak detection and topology identification in pipelines using fluid transients and artificial neural networks. Journal of Water Resources Planning and Management, 146(6), 1-11.

Chandran, J., \& Salih, A. (2019). A modified equation of state for water for a wide range of pressure and the concept of water shock tube. Fluid Phase Equilibria, 483, 182-188.

Chandran, R.J., Raju, R., \& Salih, A. (2020). An adaptively-damped compressible-liquid model for noncavitating hydraulic surges. International Journal of Engineering, 33(10), 2047-2056.

Colombo, A.F., Lee, P., \& Karney, B.W. (2009). A selective literature review of transient-based leak detection methods. Journal of hydro-environment research, 2(4), 212-227.

Covas, D., Stoianov, I., Mano, J.F., Ramos, H., Graham, N., \& Maksimovic, C. (2005). The dynamic effect of pipe-wall viscoelasticity in hydraulic transients. Part II-Model development, calibration and verification. Journal of Hydraulic Research, 43(1), 56-70. 
Covas, D., Stoianov, I., Ramos, H., Graham, N., \& Maksimovic, C. (2004). The dynamic effect of pipe-wall viscoelasticity in hydraulic transients. Part I-Experimental analysis and creep characterization. Journal of Hydraulic Research, 42(5), 517-532.

Firkowski, M., Urbanowicz, K., \& Duan, H.F. (2019). Simulation of unsteady flow in viscoelastic pipes. SN Applied Sciences, 1(6), 519.

Kodura, A. (2016). An analysis of the impact of valve closure time on the course of water hammer. Archives of Hydro-Engineering and Environmental Mechanics, 63(1), 35-45.

Lai, Z., Li, Q., Karney, B., Yang, S., Wu, D., \& Zhang, F. (2018). Numerical simulation of a check valve closure induced by pump shutdown. Journal of Hydraulic Engineering, 144(12), 06018013.

Mandair, S., Magnan, R., Morissette, J.F., \& Karney, B. (2020). Energy-based evaluation of 1D unsteady friction models for classic laminar water hammer with comparison to CFD. Journal of Hydraulic Engineering, 146(3), 04019072.

Mitosek, M., \& Szymkiewicz, R. (2012). Wave damping and smoothing in the unsteady pipe flow. Journal of Hydraulic Engineering, 138(7), 619-628.

Neuhaus, T., \& Dudlik, A. (2006). Experiments and comparing calculations on thermohydraulic pressure surges in pipes. Kerntechnik Independent Journal for Nuclear Engineering, 71(3), 87-94.

Nikpour, M.R., Nazemi, A.H., Dalir, A.H., Shoja, F., \& Varjavand, P. (2014). Experimental and numerical simulation of water hammer. Arabian Journal for Science and Engineering, 39(4), 2669-2675.

Ramos, H., Covas, D., Borga, A., \& Loureiro, D. (2004). Surge damping analysis in pipe systems: modelling and experiments. Journal of Hydraulic Research, 42(4), 413-425.

Soares, A.K., Martins, N., \& Covas, D.I. (2015). Investigation of transient vaporous cavitation: experimental and numerical analyses. Procedia Engineering, 119, 235-242.

Tran, P.D. (2017). Pressure transients caused by air-valve closure while filling pipelines. Journal of Hydraulic Engineering, 143(2), 04016082.

Wang, L., Wang, F., \& Lei, X. (2018). Investigation on friction models for simulation of pipeline filling transients. Journal of Hydraulic Research, 56(6), 888-895.

Wang, X., Lin, J., \& Ghidaoui, M.S. (2020). Usage and effect of multiple transient tests for pipeline leak detection. Journal of Water Resources Planning and Management, 146(11), 06020011.

Yang, S., Wu, D., Lai, Z., \& Du, T. (2017). Three-dimensional computational fluid dynamics simulation of valve-induced water hammer. Proceedings of the Institution of Mechanical Engineers, Part C: Journal of Mechanical Engineering Science, 231(12), 2263-2274. 